\title{
Making the invisible visible: Phase dyssynchrony has potential as a new prognostic marker
}

\author{
Kenichi Nakajima, MD, PhD, ${ }^{\mathrm{a}, \mathrm{d}}$ Koichi Okuda, PhD, ${ }^{\mathrm{b}}$ Shinro Matsuo, MD, PhD, ${ }^{\mathrm{a}}$ \\ and Piotr Slomka, $\mathrm{PhD}^{\mathrm{c}}$ \\ a Department of Nuclear Medicine, Kanazawa University Hospital, Kanazawa, Japan \\ b Department of Physics, Kanazawa Medical University, Uchinada, Japan \\ c AIM Program/Department of Imaging, Cedars-Sinai Medical Center, Los Angeles \\ d Department of Nuclear Medicine, Kanazawa University, Kanazawa, Japan
}

Received May 12, 2017; accepted May 15, 2017

doi:10.1007/s12350-017-0929-5

\section{See related article, pp. 288-297}

Quantitative nuclear imaging analysis can enhance confidence in what is visible in the images. For example, defect scoring in myocardial perfusion studies can confirm the extent and severity of perfusion abnormalities, and left ventricular ejection fraction (LVEF) can confirm the severity of cardiac contractility so that the viewpoint is universally standardized. In contrast, phase dyssynchrony analysis renders what is invisible to the naked eye visible and thus provides a viewpoint that conventional functional parameters cannot produce. Although the methodology could be very attractive, phase dyssynchrony findings derived from patients with heart disease need to be appropriately understood.

Phase analysis of the timing of ventricular contractions was initially introduced during the 1980s for gated blood-pool studies using ${ }^{99 \mathrm{~m}} \mathrm{Tc}$-red blood cells. ${ }^{1}$ Some interesting results have been generated by visualizing sequences of contraction timing derived from pixelbased or region-based time-activity curves. Studies that have shown sequences of contraction timing include bundle branch block, ${ }^{2}$ in which contraction is delayed in the electrically blocked ventricle, and pre-excitation syndrome, ${ }^{3}$ in which contraction is early at the site of an accessory conduction pathway. Regional ventricular wall motion abnormalities are measured using the two

Reprint requests: Kenichi Nakajima, MD, PhD, Department of Nuclear Medicine, Kanazawa University Hospital, 13-1 Takara-machi,

Kanazawa, 920-8641, Japan; nakajima@med.kanazawa-u.ac.jp

J Nucl Cardiol 2019;26:298-302.

$1071-3581 / \$ 34.00$

Copyright (c) 2017 American Society of Nuclear Cardiology. parameters of phase and amplitude, which show hypokinesis and dyskinesis as a very low amplitude and a delayed phase, respectively. After the advent of gated myocardial perfusion single-photon emission computed tomography (SPECT), it has been used to evaluate regional dyssynchrony and to determine the need for cardiac resynchronization therapy (CRT). ${ }^{4}$ Gated bloodpool studies use regional or pixel-based volume curves, whereas gated myocardial perfusion imaging (MPI) studies use wall thickening values or changes in counts. Current providers have recently included phase analysis in their software packages for quantitative analysis. ${ }^{4-7}$

Information about phase in gated MPI studies is a new viewpoint that differs from conventional wall motion analysis, and the role of phase analysis in myocardial damage or primary and secondary cardiomyopathy has yet to be determined. In this issue of the Journal of Nuclear Cardiology, phase analysis was applied to patients with chronic kidney disease (CKD), and increased dyssynchrony was reportedly related to prognosis. ${ }^{8}$ Before directly proceeding to clinical applications, we will review technologically confounding factors to understand the real value of phase analysis to clinical studies.

\section{PARAMETERS OF PHASE ANALYSIS}

The main parameters used for phase analysis are based on time-activity curves of regional myocardial counts, and the fundamental frequency of Fourier transformation. After creating a histogram of the phase distribution, statistical analysis including $95 \%$ bandwidth, standard deviation (SD), and entropy can be calculated. ${ }^{7,9}$ Phase values are not generally interchangeable among software programs since they depend on factors such as temporal and smoothing filters, data sampling angle, and histogram bins. Timeactivity curves are expressed using Fourier fit as follows: Direct current component + amplitude $\times \sin (x-\varphi)$, 
where phase $\varphi$ can be adjusted to the maximum point in the wall thickening curve. Some technical factors should also be considered since fitting cosine/sine functions to regional time-activity curves is influenced by the total count, amplitude, and noise. When a histogram is wide including multiple peaks, the shape is not fitted for normal distribution using SD, but $95 \%$ bandwidth or entropy could characterize the shape better, while entropy might be preferred.

\section{GENDER AFFECTS DYSSYNCHRONY}

Phase analysis of data from the Japanese Society of Nuclear Medicine working group (JSNM-WG) normal databases for gated SPECT using ${ }^{99 \mathrm{~m}}$ Tc-sestamibi or tetrofosmin ${ }^{7,10}$ identified that phase parameters (bandwidth, phase SD, and entropy) are larger in men. Although the heart is unlikely to be more dyssynchronous in males than in females, some explanations for this can be considered. If the dose is the same in men and women, which could occur when administered using a syringe-type ${ }^{99 \mathrm{~m}} \mathrm{Tc}$ imaging tracer preparation, myocardial activity (counts $/ \mathrm{cm}^{3}$ ) could be lower in men than in women, due to having a larger myocardial volume. The same filtering might result in smoother images from women than in men. From a physiological viewpoint, electrical depolarization of the heart takes longer time because the mass of the male heart is larger. Exercise intensity could be higher for men than for women, which could affect phase values.

\section{DOES HAVING REDUCED LVEF RESULT IN MORE DYSSYNCHRONY?}

The calculated bandwidth and phase SD are larger in patients with reduced LVEF. When an infarct occurs in the myocardium, the amplitude is typically low and the phase is delayed in the defective region, particularly when the region is akinetic or dyskinetic. Phase values become unstable because of the very low calculated amplitude in an akinetic myocardial segment with very low uptake. Variations in fitting could result in a larger phase bandwidth and SD. Figure 1 shows some timeactivity curves to illustrate how the noise level influences phase calculations. The SD of noise in this calculation is defined as the square root of $\mathrm{N}$ counts from Poisson statistics. A low pixel count in this simple simulation results in increased $\operatorname{Sqrt}(\mathrm{N}) / \mathrm{N}$.

\section{ARE LARGER HEARTS MORE DYSSYNCHRONOUS?}

A pathologically large left ventricular (LV) volume can coexist with low LVEF in patients with ischemic heart disease and cardiomyopathy. From a technological viewpoint, regional myocardial counts are relatively decreased in such patients due to either a larger myocardial mass or fewer accumulated myocardial counts. Regardless, myocardial counts per unit volume are reduced. From a pathophysiological viewpoint, regions of asynergy result in delayed phase, and the distribution of phase histograms is wide, or sometimes peaks are separated in the region of dyssynchrony. However, even in normal individuals in the JSNM-WG databases, ventricular volume correlates with phase bandwidth and phase SD positively and weakly, but nonetheless significantly. ${ }^{7}$

\section{MORE DYSSYNCHRONY IN THE FIRST PART OF A STRESS-REST STUDY?}

The second injection contains 2- to 4-fold more radioactivity during the one-day protocol of stress-rest studies using these tracers. This leads to more counts in the second study, meaning that more statistical noise is present in the first study. A difference in stress-rest and rest-stress protocols in MPI could affect the average values of the phase variation. Figure 2 shows the distribution of phase parameters in consecutive patients with a normal LVEF without myocardial perfusion defects at two hospitals between January and March 2017. The patients were consecutively administered with similar doses of ${ }^{99 \mathrm{~m}} \mathrm{Tc}$ sestamibi or tetrofosmin. Stress gated MPI studies at one hospital (A), where the stress-rest protocol was applied, showed a larger phase variation in the stress sequence. In contrast, studies at the other hospital (B) where the rest-stress protocol was applied, found a larger phase variation during the rest sequence. One hypothesis is that patients with threevessel disease develop dyssynchrony after stress, ${ }^{11}$ and stress studies actually show a larger phase SD and bandwidth after exercise. These findings probably reflect stress-induced ischemia or limited functional reserve in ischemic heart disease. However, they could also be due to the protocol (stress-rest or rest-stress) applied during the study. Therefore, appropriate control patients for the verification of this hypothesis are needed, and dose-related variations should also be considered.

\section{MORE DYSSYNCHRONY IN HEART DISEASES}

We would like to be able to identify dyssynchrony in a diseased heart using gated MPI. We understand that severe stress-induced ischemia in patients with coronary artery disease results in regional dyssynchrony during stress. Mori et al. studied LV dyssynchrony in ${ }^{201} \mathrm{Tl}$ perfusion images of patients with CKD. ${ }^{8}$ The incidence of major adverse cardiac events (MACE) was higher 

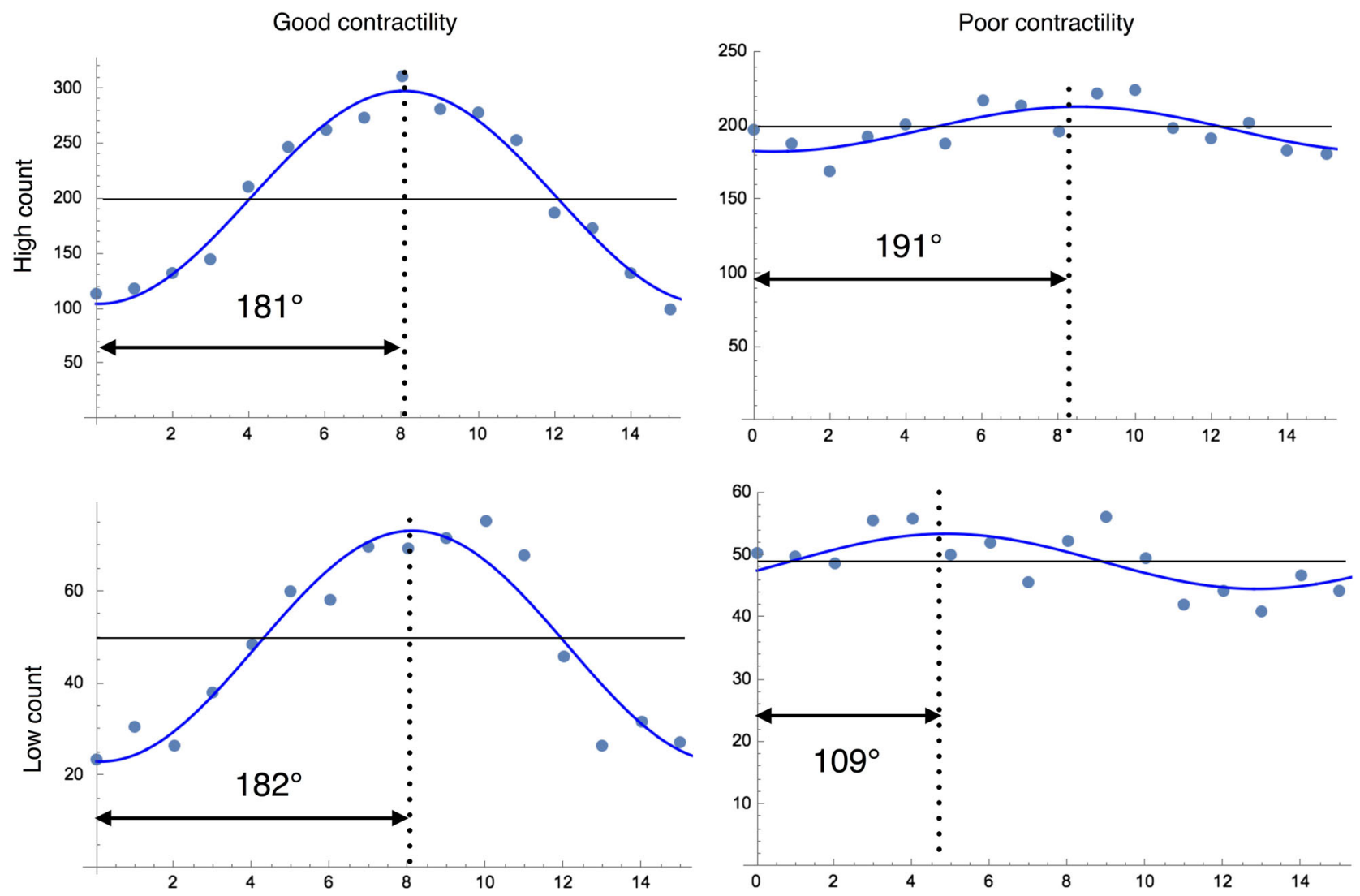

Figure 1. Simulated time-activity curves in high counts (200 count/pixel) (upper panel) and low counts $(50$ count/pixel) (lower panel) with examples of good (left) and poor (right) contractility. The (maximum - minimum)/maximum is $67 \%$ (left) and $10 \%$ (right), respectively. The original curve has a phase shift of $180^{\circ}$. In the case of poor contractility and low counts (bottom right), the computed phase values become susceptible to noise.

Hospital A: stress first

80

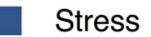

Rest (dose 2-3 times of stress)

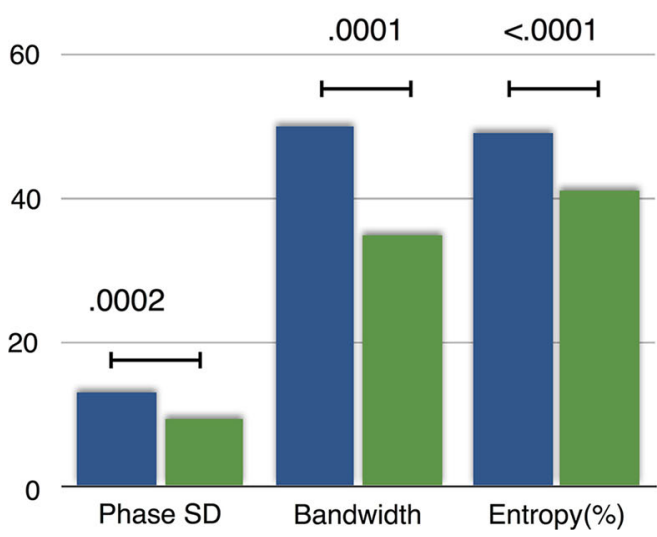

Hospital B: rest first

80

Stress (dose 2-3 times of rest)

Rest

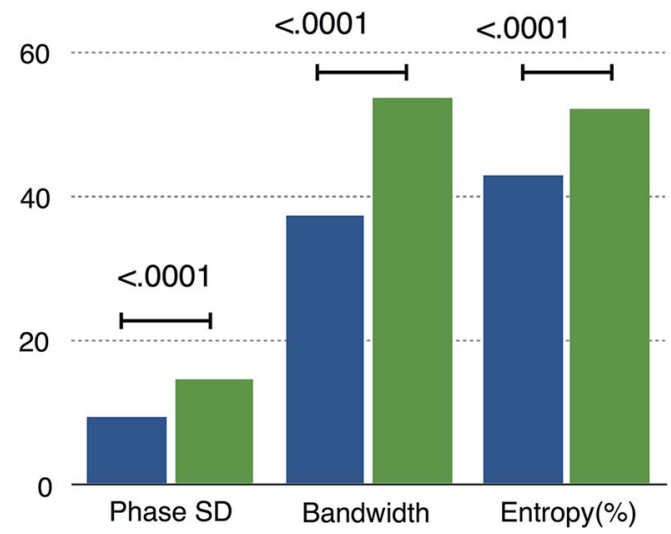

Figure 2. Phase distribution in patients with normal LVEF without perfusion abnormality at two hospitals; hospital A (stress first, $n=16$ ) and hospital B (rest first, $n=20$ ). $P$ values for the difference of stress and rest parameters are shown. Data were processed using the cardioREPO software. $^{7,12}$ 
among patients with a larger phase SD and bandwidth even in the absence of a definite perfusion defect. The QRS duration determined by electrocardiography was slightly longer in patients with MACE in agreement with dyssynchrony. LV volumes were larger, and time to end-systole was longer in the MACE group. Although ischemia might be detected more effectively using ${ }^{201} \mathrm{Tl}$, it could cause more noise than ${ }^{99 \mathrm{~m}} \mathrm{Tc}$ MPI tracers. Another study in patients with end-stage renal disease (ESRD), in which $28 \%$ received renal transplants, the phase bandwidth was greater among patients who died than among those who survived, indicating greater dyssynchrony in ESRD. ${ }^{13}$ Considering the pathophysiology of ESRD, the effects of interstitial fibrosis, microcirculatory disturbances, chronic pressure and volume overload, and epicardial coronary stenosis might exert composite effects that might cause dyssynchronous movement of the heart and result in abnormal phase patterns.

Although various factors, such as LVEF, LV volume, and perfusion defects can play roles in the prognostic analysis, the prognostic significance of phase parameters is intriguing.

Phase analysis has also been applied to CRT. ${ }^{14,15}$ Echocardiographic measurements of dyssynchrony using septal-posterior or lateral wall motion delay require that the physicians and technologists who implement the echocardiography generate reproducible results. However, one multicenter study could not identify a single specific parameter that could predict a successful outcome of CRT. ${ }^{14}$ From the perspective of technological reproducibility, nuclear studies might confer an advantage, but this should be further investigated while echocardiographic evaluation is also in progress. The LV dyssynchrony assessed from gated MPI closely correlates with dyssynchrony assessed by tissue Doppler imaging, and histogram bandwidth and phase SD correlated most closely with LV dyssynchrony. ${ }^{16}$ A combination of dyssynchrony and perfusion indices might play a role in effective CRT therapy. ${ }^{17}$ More of such comparative studies are required to confirm the effectiveness of dyssynchrony analysis.

\section{ARE PHASE ABNORMALITIES DUE TO TECHNOLOGY OR PATHOPHYSIOLOGY?}

Phase analysis could become applied more frequently in the near future for the diagnosis and prognosis of heart disease when convenient software becomes clinically available. In general, a simple hypothesis could be that more severe stages are related to more phase abnormalities. Whether phase analysis of dyssynchrony parameters provides additive information to conventional clinical parameters should be further examined. Lastly, due to dependence on software, values are not interchangeable, but Emory Cardiac Toolbox, QGS, Corridor 4DM, and cardioREPO software could be reliable tools for phase dyssynchrony analysis, ${ }^{12}$ as well as Heart Function View. ${ }^{7,8}$

Phase analysis is a potentially attractive method of evaluating dyssynchrony. However, the findings of each step of its application cannot be visually supported because they are practically invisible to the naked eye. Therefore, before proceeding to definite recommendations for the clinical application, control patients must be carefully selected, and confounding factors must be identified and excluded.

\section{Acknowledgements}

SPECT data from hospital $B$ are from Kaga Medical Center, Kaga City, Japan. The authors appreciate the editorial assistance of Norma Foster.

\section{Disclosure}

K. Nakajima collaborates with FUJIFILM RI Pharma, Tokyo, Japan, and Nihon MediPhysics, Tokyo, Japan to develop cardioREPO and Heart Function View software, respectively. K. Okuda, S. Matsuo, and P. Slomka have nothing to declare.

\section{References}

1. Links JM, Douglass KH, Wagner HN Jr. Patterns of ventricular emptying by Fourier analysis of gated blood-pool studies. J Nucl Med. 1980;21:978-82.

2. Van Kriekinge SD, Nishina H, Ohba M, Berman DS, Germano G. Automatic global and regional phase analysis from gated myocardial perfusion SPECT imaging: Application to the characterization of ventricular contraction in patients with left bundle branch block. J Nucl Med. 2008;49:1790-97.

3. Nakajima K, Bunko H, Tonami N, Taki J, Nanbu I, Shiire Y, et al. Length-based Fourier analysis in the pre-excitation syndrome. J Nucl Med. 1986;27:1131-37.

4. Chen J, Garcia EV, Folks RD, Cooke CD, Faber TL, Tauxe EL, et al. Onset of left ventricular mechanical contraction as determined by phase analysis of ECG-gated myocardial perfusion SPECT imaging: Development of a diagnostic tool for assessment of cardiac mechanical dyssynchrony. J Nucl Cardiol. 2005;12:68795.

5. Germano G, Kavanagh PB, Slomka PJ, Van Kriekinge SD, Pollard $\mathrm{G}$, Berman DS. Quantitation in gated perfusion SPECT imaging: The Cedars-Sinai approach. J Nucl Cardiol. 2007;14:433-54.

6. AlJaroudi W, Jaber WA, Grimm RA, Marwick T, Cerqueira MD. Alternative methods for the assessment of mechanical dyssynchrony using phase analysis of gated single photon emission computed tomography myocardial perfusion imaging. Int $\mathrm{J}$ Cardiovasc Imaging. 2012;28:1385-94.

7. Nakajima K, Okuda K, Matsuo S, Kiso K, Kinuya S, Garcia EV. Comparison of phase dyssynchrony analysis using gated myocardial perfusion imaging with four software programs: Based 
on the Japanese Society of Nuclear Medicine working group normal database. J Nucl Cardiol. 2016;24:611-21.

8. Mori H, Isobe S, Suzuki S, Unno K, Morimoto R, Kano N, et al. Prognostic value of left ventricular dyssynchrony evaluated by gated-SPECT in CKD patients with normal perfusion defect scores. J Nucl Cardiol. 2017. doi:10.1007/s12350-017-0889-9.

9. O'Connell JW, Schreck C, Moles M, Badwar N, DeMarco T, Olgin J, et al. A unique method by which to quantitate synchrony with equilibrium radionuclide angiography. J Nucl Cardiol. 2005; 12:441-50.

10. Nakajima K, Matsumoto N, Kasai T, Matsuo S, Kiso K, Okuda K. Normal values and standardization of parameters in nuclear cardiology: Japanese Society of Nuclear Medicine working group database. Ann Nucl Med. 2016;30:188-99.

11. Hida S, Chikamori T, Tanaka H, Igarashi Y, Shiba C, Usui Y, et al. Diagnostic value of left ventricular dyssynchrony after exercise and at rest in the detection of multivessel coronary artery disease on single-photon emission computed tomography. Circ J. 2012;76:1942-52.

12. Okuda K, Nakajima K, Matsuo S, Kashiwaya S, Yoneyama H, Shibutani T, et al. Comparison of diagnostic performance of four software packages for phase dyssynchrony analysis in gated myocardial perfusion SPECT. EJNMMI Res. 2017;7:27.
13. Aggarwal H, AlJaroudi WA, Mehta S, Mannon R, Heo J, Iskandrian AE, et al. The prognostic value of left ventricular mechanical dyssynchrony using gated myocardial perfusion imaging in patients with end-stage renal disease. J Nucl Cardiol. 2014;21:73946.

14. Chung ES, Leon AR, Tavazzi L, Sun JP, Nihoyannopoulos P, Merlino J, et al. Results of the predictors of response to CRT (prospect) trial. Circulation. 2008;117:2608-16.

15. Henneman MM, Chen J, Dibbets-Schneider P, Stokkel MP, Bleeker GB, Ypenburg C, et al. Can LV dyssynchrony as assessed with phase analysis on gated myocardial perfusion SPECT predict response to CRT? J Nucl Med. 2007;48:1104-11.

16. Henneman MM, Chen J, Ypenburg C, Dibbets P, Bleeker GB, Boersma E, et al. Phase analysis of gated myocardial perfusion single-photon emission computed tomography compared with tissue Doppler imaging for the assessment of left ventricular dyssynchrony. J Am Coll Cardiol. 2007;49:1708-14.

17. Keida T, Ohira H, Fujita M, Chinen T, Nakamura K, Kato T, et al. Quantitative assessment of dyssynchrony using ECG-gated SPECT myocardial perfusion imaging prior to and following cardiac resynchronization therapy. Circ J. 2009;73:1550-53. 